\title{
Improving Power Efficiency in WBAN Communication Using Wake Up Methods
}

\author{
Stevan Marinkovic ${ }^{1}$, Emanuel Popovici ${ }^{2}$, and Emil Jovanov ${ }^{3}$ \\ 1 ABB Corporate Research, Baden-Dättwil, Switzerland \\ stevan.marinkovic@ch.abb.com \\ 2 University College Cork, Cork, Ireland \\ e.popovici@ucc.ie \\ 3 The University of Alabama, Huntsville, USA \\ emil.jovanov@uah.edu
}

\begin{abstract}
Power efficient communication in a Wireless Body Area Network (WBAN) is critical for successful system deployment. Stringent constraints of size and weight of sensors significantly limit available sensor power, particularly in the case of implantable sensors. This paper discusses and analyses methods that could be used to improve power efficiency of implantable WBAN systems, with focus on the Wake Up Radio (WUR), which allows power efficient listening of wireless channel. The paper presents analysis of existing hardware and design trade offs in WUR implementation.
\end{abstract}

Key words: Wireless Body Area Networks, Wake-up Radio, Ultra Low Power

\section{Introduction}

Wireless Body Area Networks (WBAN) have witnessed a surge of resurgence in research and applications in recent years $[1,2,3,4]$. This trend is facilitated by advances in sensing, processing and communication algorithms and technologies. WBANs integrate a number of wearable or implanted sensors and may integrate environmental sensors in the vicinity of the user. Environmental sensors typically provide context of physiological or activity records. User convenience and ubiquitous connectivity, among other advantages, make WBANs the most promising application of wireless sensor networks. WBAN applications have the potential to dramatically change the field of monitoring, telemedicine, drug delivery and compliance monitoring, as well as computer assisted rehabilitation.

WBAN based systems introduce a very specific design space with stringent constraints of weight and size, resulting in significant resource constraints. The most limiting factor is power source, which determines maximum power consumption of the system. This is particularly important for implanted sensors, such as pacemakers, where battery life is expected to exceed 10 years [5]. Wireless communication is typically dominant component in the power budget, with several times higher power consumption than processing in the active mode. This 
paper presents a survey of power efficient WBAN protocols and architectures, with emphasis on benefits of integration of Wake Up Radio as resource that facilitates power efficient WBAN communication.

\section{Communication in WBAN}

Due to the power constraints, a typical WBAN implements a simple network topology, while supporting sophisticated protocols and signal processing algorithms as well as high quality of service required by health monitoring systems. This section presents commonly used wireless communication standards and system support for power efficient wireless communication.

\subsection{Standard Wireless Communication}

Choice of wireless standard is mostly driven by requirements for power efficiency and system support. Consequently, most research projects employ IEEE 802.15.1 (Bluetooth [6]) or IEEE 802.15.4 (ZigBee [7]) protocols. These wireless standards are well researched, documented and tested, but are not an optimal technologies for the wireless BAN, since they target a large application space, more flexible networks than WBAN and are used for longer transmission ranges [8]. This makes them less energy efficient than the protocols specifically targeting WBANs such as [9]. A comparison and optimization of two popular WBAN technologies, Bluetooth and ZigBee, is given in the comparative study [10], in terms of design, cost, performance and energy efficiency. Demand for low power consumption, essential for WBAN applications led to the modification of the original Bluetooth standard and introduction of Bluetooth Low Energy (LE). Figure 1 shows the typical WBAN implementation. Smartphone serves as a master node controlling the WBAN and gateway for integration of data from wearable or implanted sensors

The long term health monitoring of patients requires low power techniques [11]. Medium Access Control (MAC) protocols play a significant role in determining the energy consumption in wireless communication. Traditional MAC protocols mainly focus on improving bandwidth utilization, throughput, and latency. However, they lack energy conserving mechanisms, which is one of the most important constraints of a WBAN. The main sources of energy waste are collision, idle listening, overhearing, and control packet overhead. MAC protocols maximize the network lifetime by controlling the aforementioned sources of energy waste. Some contention based protocols such as WiseMAC [12], BMAC [13] and [14] use low power listening and preamble sampling techniques to reduce idle listening. Other protocols such as SMAC [15], TMAC [16] and PMAC [17] reduce idle listening by applying a synchronized schedule between the nodes. Contention-based solutions are not suitable for WBAN since most of the traffic is correlated. 


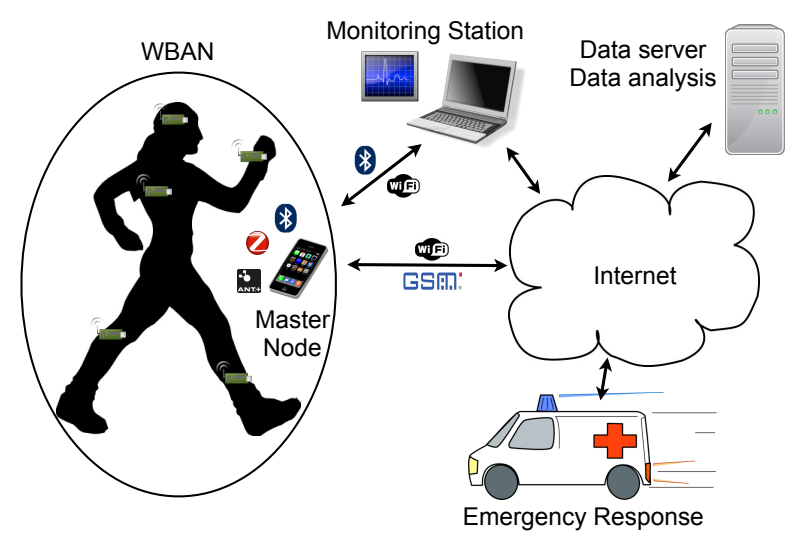

Fig. 1. Wireless Body Area Network example

Some research groups used TDMA-based MAC protocols for wireless sensor networks $[18,19,20]$ and protocols especially for WBAN [21, 22, 23, 24]. Some protocols are designed to support application specific transceivers [21, 22].

The main schemes for MAC protocols for sensor networks are CSMA/CA (Carrier Sense Multiple Access / Collision Avoidance) and TDMA (Time Division Multiple Access). FDMA (Frequency Division Multiple Access) requires complex hardware and CDMA (Code Division Multiple Access) has high computational demands. A comparison between TDMA and CSMA protocols is given in Table 1, as reported in [25], where is shown that the TDMA method is more suitable for static types of networks. It is presumed that a BAN is not a dynamic network structure.

Table 1. Comparison of TDMA and CSMA/CA

\begin{tabular}{|l|l|l|}
\hline Communication Strategy & TDMA & CSMA/CA \\
\hline \hline Power Consumption & Low & High \\
\hline Bandwidth Utilisation & Maximum & Low \\
\hline Preferred Traffic Level & High & Low \\
\hline Dynamic (Network Change) & Poor & Good \\
\hline Effect of Packet Failure & Latency & Low \\
\hline Synchronisation & Essential & - \\
\hline
\end{tabular}

TDMA based protocols outperform CSMA based protocols in all areas except the protocol adaptability to changes in network topology. On the other hand TDMA based protocols need a good synchronization scheme. Such schemes are not easy to implement in a dynamic network, but since BAN has relatively 
constant network structure and fixed sensor functions synchronization can be simplified.

Additionally, most of the contention-based protocols use Clear Channel Assessment (CCA) to determine the status of the channel. However, this is not always guaranteed in WBAN due to the high path loss inside or outside the human body. Schedule-based protocols such as Time Division Multiple Access (TDMA) provide good solutions to the traffic correlation and CCA problems. These protocols are energy conserving protocols because the duty cycle is reduced and there is no contention, idle listening, and overhearing problem. However, common TDMA needs extra energy for periodic time synchronization. All the sensors (with and without data) are required to receive periodic control packets in order to synchronize their clocks.

\subsection{Preamble Sampling}

Another method for reducing time spent on idle listening is called preamble sampling method. This method is based on the principle of short duration of radio activity, to determine if there is activity on the channel, in order to wake up. Sensor node wakes up periodically, and if the channel is busy the node remains awake; if not, the node returns to sleep. Time between two channel samples is fixed and known by the network. Therefore, in order to wake up the nodes, before transmission one must ensure that the preamble before the transmission is long enough (at least as long as this time between the channel sampling).

Table 2. Summary of preamble sampling methods [26]

\begin{tabular}{|l|l|l|l|l|}
\hline Category & Energy & Sync. Requirements & Implementation & Adaptability \\
\hline \hline Basic preamble sampling & Average & None & Simple & Bad \\
\hline Short preamble burst & Good & None & Simple & Bad \\
\hline Sync. info. advantage & Very good & Sync. needed & Average & Bad \\
\hline Adaptive duty cycle & Good & None & Not simple & Good \\
\hline
\end{tabular}

As summarised in Cano et.al. [26], Preamble sampling techniques can be split into the following four 4 categories:

1. Basic preamble sampling. - This technique is first presented in 2002 [27], where it was implemented in Aloha and called "preamble sampling". Around same time it was presented in [28], where it was combined with CSMA and called "low power listening". Later, Berkeley MAC was presented [13], which had clear channel assessment (CSA) strategy, where noise floor was measured in the period where it was known that there is no communication on the channel. 
2. Short preamble burst. - This method is a bit different from the previous in a sense that long preamble is split into a series of short packets. In such way, preamble sampling can detect channel activity and read a short packet of data that will most commonly be a short address (destination identifier), allowing to target specific node. In this way, unnecessary listening is avoided. Example of these are Enhanced B-MAC [29]. Also, one can include information when will the data transmission start, allowing the node to return to sleep mode for a while [13, 30, 31, 32, 33]. Further improvements of this method can be: The transmission of early acknowledgement, to stop the transmission of the burst if the node has nothing to transmit, as presented in $[34,35,36,31,37]$ and others; Repetitions of data packet, where full data message is repeated [30]; or packet dependent behaviour, that depends on the type of the packet being sent $[38,31,39]$.

3. Taking advantage of synchronisation information. - This preamble sampling category is a combination of preamble sampling and synchronisation mechanism, with the goal of reducing the length of the preamble. There are two methods of achieving this, either the network coordinator memorises the wake-up time of each receiver, and positions its preamble at that time $[12,35,32,40]$; or wake up schedules of sensors are synchronised [41, 39].

4. Adaptive duty cycle. - This technique is based on changing of the duty cycle of the preamble sampling, channel listening, to adapt to the network traffic change. This makes the protocol to be better adaptive to the network changes, and traffic intensity change, without much idle listening but requires a more complicated algorithm to run. This duty cycle change can be either request based, where duty cycle is adapted on the requests in transmission $[12,42,43,44]$, or traffic load based, where the duty cycle is changed with the observed traffic load $[45,46,47,41,36]$. A survey and comparison of WUR methods is presented in [26] and outlined in Table 2.

\subsection{Wake Up Radio}

The problem of idle listening for events can be solved using the separate hardware that is continuously listening for a certain wake up signal. Typical application will be in a WBAN network with ranges less than $2 \mathrm{~m}$. There will be a dedicated device acting as a Master Node that will act as a network coordinator. This device would send wake up signals, and receive data packets from sensors. In 2001, [48] presented the advantages of wake up radio and estimated that a specialised radio interface could consume as little energy as $1 \mu \mathrm{W}$.

The wake up receiver intended for WBAN is expected to operate in the dense network environment. At any given moment some nodes will be communicating within a WBAN, while some may stay in a sleep mode, monitoring the channel for communication requests. Also, we can expect numerous high power transceivers and noise in the vicinity of a network. The optimal WUR must be immune to to this ambient traffic and should avoid waking up the sensor on signals intended for the neighbouring nodes, as well as on the ambient noise. 
From a functional perspective, WUR is not as restricted regarding bit error rate and data rate as the standard receiver. The metric of interest is probability of detection of wake up signals and probability of false alarms. Retransmissions increase power consumption for the transmitter as well as the latency. False alarms are costly from a power perspective because of the needless sensor activations.

There are two functional groups of WUR: wake up circuits and wake up receivers.

Wake up circuits can have very low power consumption (even zero power), but these only detect the activity on a channel, and cannot distinguish a wake up signal from other RF activity of sufficient power. They are mostly realised using a charge pump. This concept was first presented and simulated in [49]. The circuit is zero power, and it is realised using the Schottky diodes. A similar solution using MOSFET is presented in [50]. Also, battery assisted (semi passive) RFID tags demand similar solutions for their power-on sensing circuitry as explained in [51]. In 1998 an RF field detector was presented as a low power wake up method that could be used in semi passive RFID tags [52]. Another design, with a poweron circuit based on multi stage charge pump is presented in [53]. A complete micro-power sensor node with RF quasi-passive wake-up circuit is presented in [54].

Wake up receivers (WUR) have the ability to demodulate and decode some packet of information following the signal, which can be used for addressing to reduce the number of activations. A low power WUR that has addressing capabilities is presented by [55]. A solution with a Schottky voltage doubler followed by a programmable amplifier and integrator is developed in [56]. Alternative approach based on a zero-bias Schottky voltage doubler (charge pump, envelope detector), is simulated in [57]. A design based solely on amplifiers is presented in [58]. Another low voltage - low power ASIC solution is presented in [59]. This WUR has a very good sensitivity of $-71 \mathrm{dBm}$. [60] proposes a WUR that has a dedicated low power microcontroller for packet decoding. The solution published in [61] is a wake-up transceiver, meaning that a dedicated transmitter was developed with the WUR. [62] have developed a low-power, low-frequency WUR with an integrated correlator which compares the received signal to a byte pattern saved in a configuration register. Another integrated solution has been developed at Fraunhofer institute [63].

Currently, the lowest power WUR, specially designed for the WBAN and implant communication is presented in [64]. This receiver is a full WUR solution, working in $400 \mathrm{MHz}$ range, that continuously listens on a wireless channel for the Wake Up Packet - a packet of OOK data that contains the address of the targeted device. Any transmitter that can work in OOK mode can be used for the transmission of the wake up packet. Ideally, one would use a transmitter that has a OOK and FSK mode, where OOK would be used for the wake up, and FSK for the regular communication. Example can be Texas Instruments CC430, 
Table 3. Wake-up receiver prototypes

\begin{tabular}{|l|c|c|c|c|c|}
\hline Authors & Year & $\mathrm{f}[\mathrm{MHz}]$ & Rate $[\mathrm{kb} / \mathrm{s}]$ & $\mathrm{S}[\mathrm{dBm}]$ & $\mathrm{P}[\mu \mathrm{W}]$ \\
\hline \hline Pletcher [58] & 2009 & 2000 & 100 & -72 & 52 \\
\hline Fraunhofer [63] & 2010 & 868 & 1 & -60 & 33 \\
\hline Le-Huy [57] & 2008 & 2400 & 50 & -50 & 20 \\
\hline Durante [56] & 2009 & 2400 & 100 & -53 & 12.5 \\
\hline Gamm [62] & 2010 & 868 & NA & -52 & 2.78 \\
\hline Doorn [60] & 2009 & 868 & 0.862 & -51 & 2.6 \\
\hline Ansari [55] & 2009 & 868 & 0.75 & N/A & 2.6 \\
\hline Hambeck [59] & 2011 & 868 & 100 & -71 & 2.4 \\
\hline Marinkovic [64] & 2011 & 434 & 5.5 & -51 & 0.270 \\
\hline
\end{tabular}

which can provide a full solution with the processor for protocol and wake-up handling, and transceiver for the communication that can be used for wake up packet transmission in OOK mode. It is currently the WUR prototype with the lowest power consumption and very low latency that was achieved at the cost of somewhat reduced sensitivity optimized for typical WBAN ranges.

Finally, Zarlink [65] present and produce a full commercial solution of wake up receiver and a transceiver, intended for implant communication. This is not a continuous listening WUR, but separate low power $2.4 \mathrm{GHz}$ receiver that is duty cycled, acting as a WUR. Reason is that this band allows for higher output power than the MICS band [66] and somewhat longer range. Although this is in a sense a preamble sampling mechanism, it is a separate circuit with a very low power consumption, and can be considered as a Wake Up Receiver.

Jelicic et.al. [67] finely summarises the state of the art in WUR designs and prototypes (Table 3) for the last three years. The paper also contains more detailed analysis of the WUR implementation in communication protocols. For the implant communication, one should consider the lower power WUR solutions in the $1 \mu \mathrm{W}$ range. Two solutions, [65] and [64] have reduced the power consumption below $1 \mu \mathrm{W}$, which makes them suitable for the implant communication. They both have advantages and disadvantages, which will be discussed in the next section.

\section{Power efficiency with WUR}

The main issue in low power wireless networking is to keep the network functionality, but to lower the power consumption. This is achieved by deliberately increasing the communication latency, to reduce the communication duty cycle. Most of the proposed and cited protocols are based around a centralised network structure and beacon communication. This means that either the network coordinator sends beacons at regular time intervals, or the sensor scans the channel at regular time intervals, in order to keep the network alive, and to synchronise for data transfer. Since data transfer cannot happen in those protocols before 
Table 4. Comparison between communication methods

\begin{tabular}{|l|l|l|l|}
\hline Communication method & Node responsiveness $\dagger$ & Range & Power consumption $\ddagger$ \\
\hline \hline Standard Protocol & Slow & High & High \\
\hline Preamble Sampling & Slow & High & Medium \\
\hline Strobed WUR (Zarlink) & Fast & Medium & Low \\
\hline WUR & Very fast & Low & Low \\
\hline$\dagger$ - Comparison with given power consumption target \\
$\ddagger$ - Comparison with given latency / response time target
\end{tabular}

the initial polling (reception of the beacon and request for data send/receive), the maximum latency is the time interval between two beacons.

When the communication is frequent, this is proper way to organise a network, since a timeslot can be given and data can be sent during regular time intervals, but when communication is scarce, or irregular, this regular awakening becomes the main communication power consumer, especially when desired latency is low. It is not mentioned in the literature, but in practice, this kind of operation requires an active timing and control circuit, which if not implemented within the transceiver, is usually a microcontroller that consumes current by staying in active mode or running the timers.

The WUR can be used on top of the existing protocols, as a method to control the power consumption, and solve the latency problem, by allowing the rest of the circuit to be in the low power "sleep" mode, while continuously listening to a wireless channel. The next sections discuss the usage of the proposed WUR, and its benefits in the standardised and frequently used WBAN protocols.

Introducing the wake up receiver as a component that can listen to a channel reduces the duty cycle (time spent on idle listening), but introduces new component with quiescent power consumption. Good power model must be derived to justify the introduction of this component and maximum power consumption it can have in order to be practical in applications.

Table 4 presents the functional comparison between communication methods in WBAN. The main point is that WUR enabled devices will have lower power consumption while maintaining the lower node response times (fast responsiveness). Of course this is achieved at the cost of the receiver sensitivity, and limitation of the effective communication range. Therefore, since the ranges in WBAN are not long and power consumption requirements are strict, one can conclude that WUR is the good approach in the implant communication, where responsiveness has to be relatively fast.

In order to determine the power consumption benefits, usage of WUR will be compared to three popular protocols. Zigbee and Bluetooth Low Power as the standard protocols, and Wise MAC as preamble sampling protocol.

ZigBee is a specification for a suite of high level communication protocols using small, low-power digital radios based on the IEEE 802.15.4-2003 standard for Low-Rate Wireless Personal Area Networks (LR-WPANs), such as wireless light switches with lamps, electrical meters with in-home-displays, consumer 
electronics equipment via short-range radio needing low rates of data transfer. The technology defined by the ZigBee specification is intended to be simpler and less expensive than other WPANs, such as Bluetooth. ZigBee is targeted at $\mathrm{RF}$ applications that require a low data rate, long battery life, and secure networking [7].

Bluetooth Low Energy (LE) is one of the most popular WBAN protocols, and it is used today for the variety of applications. It uses a TDMA based master-slave protocol, consisting of piconets with one master and up to 7 slaves [68]. Bluetooth channels use a Frequency-Hop/Time-Division-Duplex (FH/TDD) scheme in which the time is divided into $625 \mu$ sec intervals called slots. The master-to-slave transmission starts in even numbered slots, while the slave-to-master transmission starts in odd-numbered slots.

The WiseMAC [12] is a popular low power MAC protocol, based on preamble sampling which is discussed for the next standard in the 802.15 CSEM FM-UWB Proposal [69] for wide band personal area networks. WiseMAC is based on the preamble sampling technique which consists in regularly sampling the medium to check for activity. All sensor nodes in a network sample the medium with the same constant period TW but their relative sampling schedule offsets are independent and constant. If the medium is found busy, a sensor node continues to listen until a data frame is received or until the medium becomes idle again. At the access point, a wake-up preamble of size equal to the sampling period is transmitted as a preamble of every data frame to ensure that the receiver will be awake when the data portion of the packet arrives. The access point (coordinator node) knows the sampling schedule of all sensor nodes, therefore it starts the transmission just at the right time with a wake-up preamble. This wake up preamble is received with the conventional receiver. The access point is the only initiator of the communications.

However losses in the body signal propagation are considerable despite the fact that WBAN implant communication ranges are low. Therefore one has to keep balance between power consumption, receiver sensitivity, and responsiveness. Depending on the application, one can choose strobed WUR (Higher range, slower response) or continuous WUR (Lower range, faster response). Both methods are much more power efficient than regular communication, or preamble sampling using the high sensitivity receiver.

Figure 2 compares the power consumption of three protocols, Zigbee, Bluetooth LE and WiseMAC, for connection events only - wireless overhead communication that is required just to keep the network alive and not to transmit or receive any data. Values for calculation was extracted from application note [70] for Bluetooth LE, application note [71] for Zigbee and [12] for WiseMAC. Data from these papers was used to calculate the energy needed for one connection event. The figure presents the average power consumption of these connection events, as a function of their frequency (time between them). In order to compare, the line "With WUR" shows the power consumption of a receiver and $\mathrm{uC}$ processor sleep mode plus the power consumption of WUR presented in [64]. This 


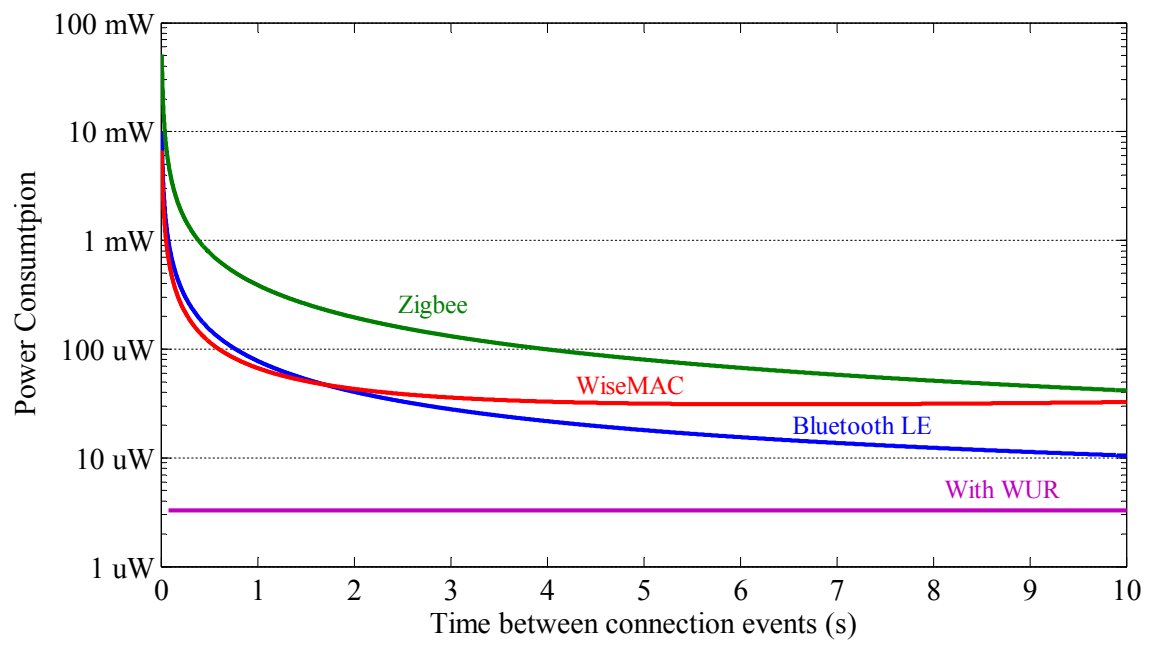

Fig. 2. Comparison between Zigbee, Bluetooth LE, WiseMAC and a protocol that would implement WUR.

is not a function of connection events frequency, as the protocol implementing WUR would not use connection events.

There are not many protocols that consider the WUR hardware in the MAC design, and this area is currently being researched. Examples are traffic-adaptive MAC protocol (TaMAC) for WBAN that improves energy efficiency by exploiting the traffic information (traffic-patterns) of the nodes is presented in [11]. Other solutions that incorporate WUR for energy efficiency is presented in $[72,55]$. These solutions are based on a fictional (concept) WUR, but prove that if presented with a WUR device, the power consumption of any protocol can be significantly reduced. [73] proposes a communication method for medical oriented scenarios using the WUR [64].

\section{Conclusion}

Efficient integration of implanted sensors into WBANs must provide effective trade off between low power consumption and the responsiveness of the implanted node to external requests. Keeping responsiveness using traditional wireless method of connection events is relatively power consuming, even in the case of low power protocols. Therefore, one should consider the new technologies of wake up receivers used for channel monitoring. In this paper we presented alternative approaches, the preamble sampling WUR by Zarlink and continuous channel listening as state of the art in this area and as seen in Figure 2, this might be a possible and promising technology for future WBAN development. 


\section{References}

[1] Istepanian, R., Jovanov, E., Zhang, Y.: Guest editorial introduction to the special section on M-Health: beyond seamless mobility and global wireless Health-Care connectivity. IEEE Transactions on Information Technology in Biomedicine, 8(4), 405-414 (2004)

[2] Ullah, S., Higgins, H., Braem, B., Latre, B., Blondia, C., Moerman, I., Saleem, S., Rahman, Z., Kwak, K.: A comprehensive survey of wireless body area networks. Journal of Medical Systems pp. 1-30 (2010)

[3] Milosevic, M., Shrove, M., Jovanov, E.: Applications of smartphones for ubiquitous health monitoring and wellbeing management. JITA 1(1), 7-15 (2011)

[4] Jovanov, E., Milenkovic, A.: Body area networks for ubiquitous healthcare applications: Opportunities and challenges. J Med Syst (2011)

[5] Shi, W.V., Zhou, M.: Body sensors applied in pacemakers: A survey. IEEE Sensors Journal 12(6), 1817-1827 (2012)

[6] Bluetooth SIG: Specification of the bluetooth system, version 4.0 (2010)

[7] ZigBee Alliance: Zigbee wireless standard (2003), http://www.zigbee.org/ Standards/Overview . aspx

[8] Drude, S.: Requirements and application scenarios for body area networks. Mobile and Wireless Communications Summit 16th IST pp. 1-5 (2007)

[9] Marinkovic, S.J., Popovici, E.M., Spagnol, C., Faul, S., Marnane, W.P.: Energyefficient low duty cycle MAC protocol for wireless body area networks. IEEE transactions on information technology in biomedicine 13(6), 915-925 (2009)

[10] Yan, L., Zhong, L., Jha, N.: Energy comparison and optimization of wireless bodyarea network technologies. Proc. Int'l Conf. Body Area Networks, BodyNets pp. 1-8 (2007)

[11] Ullah, S., Kwak, K.S.: An ultra low-power and traffic-adaptive medium access control protocol for wireless body area network. Journal of Medical Systems pp. $1-10(2010)$

[12] Hoiydi, E., Decotignie, J.: Wisemac: An ultra low power mac protocol for the downlink of infrastructure wsns. Ninth International Symposium on Computers and Communications (ISCC) pp. $244-251$ (2004)

[13] Polastre, J., Hill, J., Culler, D.: Versatile low power media access for wireless sensor networks. 2nd International Conference on Embedded Networked Sensor Systems pp. 95-107 (2004)

[14] Hauer, J., Handziski, V., Köpke, A., Willig, A., Wolisz, .A.: A component framework for content-based publish/subscribe in sensor networks. Proceedings of the 5th European conference on Wireless sensor networks pp. 369-385 (2008)

[15] Wei, Y., Heidemann, J., Estrin, D.: An energy-efficient mac protocol for wireless sensor networks. IEEE Infocom conference pp. 1567 - 1576 (2002)

[16] van Dam, T., Langendoen, K.: An adaptive energy-efficient mac protocol for wsns. 1st ACM Conf. on Embedded Networked Sensor Systems (SenSys) pp. 171-180 (2003)

[17] Khan, N., Boncelet, C.: Pmac: Energy efficient medium access control protocol for wireless sensor networks. IEEE Military Communications Conference pp. 1-5 (2006)

[18] Lee, W., Datta, A., Cardell-Oliver, R.: Fleximac: A flexible tdma-based mac protocol for fault-tolerant and energy-efficient wireless sensor networks. 14th IEEE Int'l Conf. Networks (ICON) pp. 1-6 (2006) 
[19] Elsaify, A., Padhy, P., Martinez, K., Zou, G.: Gwmac- a tdma based mac protocol for a glacial sensor network. 4th ACM PE-WASUN pp. 54-61 (2007)

[20] van Hoeselt, L., Niebergt, T., H.J.Kipt, Havingar, P.: Advantages of a tdma based, energy-efficient, self-organizing mac protocol for wsns. IEEE 59th Vehicular Technology Conference 3, 1598 - 1602 (2004)

[21] Chen, Z., Khokhar, A.: Self organization and energy efficient TDMA MAC protocol by wake up for wireless sensor networks. First Annual IEEE Communications Society Conference on Sensor and Ad Hoc Communications and Networks, 2004. pp. 335-341 (2004)

[22] Omeni, O., Wong, A., Burdett, A., Toumazou, C.: Energy efficient medium access protocol for wireless medical body area sensor networks. IEEE Transactions on Biomedical Circuits and Systems 2, 251-259 (2007)

[23] Milenkovic, A., Otto, C., Jovanov, E.: Wireless sensor network for personal health monitoring: issues and an implementation. Computer Communications 29, 2521$2533(2006)$

[24] Latre, B., Braem, B., Moerman, I., Blondia, C., Reusens, E., Joseph, W., Demeester, P.: A low-delay protocol for multi-hop wireless body area networks. 4th Int'l Conference, MobiQuitous pp. 1-8 (2007)

[25] Cionca, V., Newe, T., Dadarlat, V.: Tdma protocol requirements for wireless sensor networks. 2-nd Int'l Conf, Sensorcomm pp. 30-35 (2008)

[26] Cano, C., Bellalta, B., Sfairopoulou, A., Oliver, M.: Low energy operation in wsns: A survey of preamble sampling mac protocols. Computer Networks 55(15), 33513363 (2011)

[27] El-Hoiydi, A.: Aloha with preamble sampling for sporadic traffic in ad hoc wireless sensor networks. In: Communications, 2002. ICC 2002. IEEE International Conference on. vol. 5, pp. 3418-3423. IEEE (2002)

[28] Hill, J., Culler, D.: Mica: A wireless platform for deeply embedded networks. Micro, IEEE 22(6), 12-24 (2002)

[29] Lim, S., Kim, S., Cho, J., An, S.: Medium access control with an energy-efficient algorithm for wireless sensor networks. In: Personal Wireless Communications. pp. 334-343. Springer (2006)

[30] Wong, K., Arvind, D.: Speckmac: low-power decentralised mac protocols for low data rate transmissions in specknets. In: Proceedings of the 2nd international workshop on Multi-hop ad hoc networks: from theory to reality. pp. 71-78. ACM (2006)

[31] Lim, S., Ji, Y., Cho, J., An, S.: An ultra low power medium access control protocol with the divided preamble sampling. Ubiquitous Computing Systems pp. 210-224 (2006)

[32] Shi, X., Stromberg, G.: Syncwuf: An ultra low-power mac protocol for wireless sensor networks. Mobile Computing, IEEE Transactions on 6(1), 115-125 (2007)

[33] Han, K., Lim, S., Lee, S., Lee, J., An, S.: Signaling-embedded short preamble mac for multihop wireless sensor networks. Information Networking. Towards Ubiquitous Networking and Services pp. 1-10 (2008)

[34] Lin, E., Rabaey, J., Wolisz, A.: Power-efficient rendez-vous schemes for dense wireless sensor networks. In: Communications, 2004 IEEE International Conference on. vol. 7, pp. 3769-3776. IEEE (2004)

[35] Mahlknecht, S., Bock, M.: Csma-mps: A minimum preamble sampling mac protocol for low power wireless sensor networks. In: Factory Communication Systems, 2004. Proceedings. 2004 IEEE International Workshop on. pp. 73-80. IEEE (2004) 
[36] Buettner, M., Yee, G., Anderson, E., Han, R.: X-mac: a short preamble mac protocol for duty-cycled wireless sensor networks. In: Proceedings of the 4th international conference on Embedded networked sensor systems. pp. 307-320. ACM (2006)

[37] Liu, S., Fan, K., Sinha, P.: Cmac: an energy-efficient mac layer protocol using convergent packet forwarding for wireless sensor networks. ACM Transactions on Sensor Networks (TOSN) 5(4), 29 (2009)

[38] Bernardo, L., Oliveira, R., Pereira, M., Macedo, M., Pinto, P.: A wireless sensor mac protocol for bursty data traffic. In: Personal, Indoor and Mobile Radio Communications, 2007. PIMRC 2007. IEEE 18th International Symposium on. pp. 1-5. IEEE (2007)

[39] Merlin, C., Heinzelman, W.: Schedule adaptation of low-power-listening protocols for wireless sensor networks. Mobile Computing, IEEE Transactions on 9(5), 672$685(2010)$

[40] Zhang, X., Ansari, J., Mähönen, P.: Traffic aware medium access control protocol for wireless sensor networks. In: Proceedings of the 7th ACM international symposium on Mobility management and wireless access. pp. 140-148. ACM (2009)

[41] Ye, W., Silva, F., Heidemann, J.: Ultra-low duty cycle mac with scheduled channel polling pp. 321-334 (2006)

[42] Anwander, M., Wagenknecht, G., Braun, T., Dolfus, K.: Beam: A burst-aware energy-efficient adaptive mac protocol for wireless sensor networks. In: International Conference on Networked Sensing Systems (INSS) (2010)

[43] Bing, L., Lin, Z., Huimin, Z.: An adaptive schedule medium access control for wireless sensor networks. In: Networking, 2007. ICN'07. Sixth International Conference on. pp. 12-12. IEEE (2007)

[44] Kumar, P., Gunes, M., Mushtaq, Q., Blywis, B.: A real-time and energy-efficient mac protocol for wireless sensor networks. International Journal of Ultra Wideband Communications and Systems 1(2), 128-142 (2009)

[45] Avvenuti, M., Vecchio, A.: Adaptability in the b-mac+ protocol. In: Parallel and Distributed Processing with Applications, 2008. ISPA'08. International Symposium on. pp. 946-951. IEEE (2008)

[46] Hurni, P., Braun, T.: Maxmac: A maximally traffic-adaptive mac protocol for wireless sensor networks. Wireless Sensor Networks pp. 289-305 (2010)

[47] Cano, C., Bellalta, B., Sfairopoulou, A., Barceló, J.: A low power listening mac with scheduled wake up after transmissions for wsns. Communications Letters, IEEE 13(4), 221-223 (2009)

[48] da Silva Jr, J.L., Shamberger, J., Ammer, M.J., Guo, C., Li, S., Shah, R., Tuan, T., Sheets, M., Rabaey, J.M., Nikolic, B., et al.: Design methodology for PicoRadio networks. Proceedings of the conference on Design, automation and test in Europe pp. 314-325 (2001)

[49] Gu, L., Stankovic, J.A.: Radio-triggered wake-up for wireless sensor networks. Real-Time Systems 29(2), 157-182 (2005)

[50] Kim, H., Cho, H., Xi, Y., Kim, M., Kwon, S., Lim, J., Yang, Y.: CMOS passive wake-up circuit for sensor network applications. Microwave and Optical Technology Letters 52, 597-600 (2010)

[51] Protocols, E.R.F.I.: Uhf rfid protocol for communications at $860 \mathrm{mhz} 960 \mathrm{mhz}$, version $1.0 .9(2005)$

[52] Lee, M.: Zero-bias detector yields high sensitivity with nanopower consumption. Linear Technology Magazine 8(1), 28 (1998) 
[53] Wenyi, C., Shuo, G., Xiao, W., Tingwen, X., Jingtian, X., Xi, T., Na, Y., Hao, M.: Analysis and design of power efficient semi-passive RFID tag. Journal of Semiconductors 31(7), 075013 (2010)

[54] Malinowski, M., Moskwa, M., Feldmeier, M., Laibowitz, M., Paradiso, J.A.: CargoNet: a low-cost micropower sensor node exploiting quasi-passive wakeup for adaptive asynchronous monitoring of exceptional events. Proceedings of the 5th international conference on Embedded networked sensor systems (SenSys) pp. 145-159 (2007)

[55] Ansari, J., Pankin, D., Mahonen, P.: Radio-triggered wake-ups with addressing capabilities for extremely low power sensor network applications. International Journal of Wireless Information Networks 16(3), 118-130 (2009)

[56] Durante, M.S., Mahlknecht, S.: An ultra low power wakeup receiver for wireless sensor nodes. Proceedings of the Third International Conference on Sensor Technologies and Applications (SENSORCOMM) pp. 167-170 (2009)

[57] Le-Huy, P., Roy, S.: Low-Power Wake-Up radio for wireless sensor networks. Mobile Networks and Applications pp. 1-11 (2008)

[58] Pletcher, N., Gambini, S., Rabaey, J.: A $52 \mu \mathrm{W}$ wake-up receiver with -72 dbm sensitivity using an uncertain-IF architecture. IEEE Journal Of Solid-State Circuits 44(1), 269-280 (2009)

[59] Hambeck, C., Mahlknecht, S., Herndl, T.: A $2.4 \mu \mathrm{w}$ wake-up receiver for wireless sensor nodes with- 71dbm sensitivity. In: Circuits and Systems (ISCAS), 2011 IEEE International Symposium on. pp. 534-537. IEEE (2011)

[60] der Doorn, B.V., Kavelaars, W., Langendoen, K.: A prototype Low-Cost wakeup radio for the $868 \mathrm{MHz}$ band. Int. Journal of Sensor Networks 5(1), 22-32 (2009)

[61] Van Langevelde, R., Van Elzakker, M., Van Goor, D., Termeer, H., Moss, J., Davie, A.: An ultra-low-power 868/915 mhz rf transceiver for wireless sensor network applications. In: Radio Frequency Integrated Circuits Symposium, 2009. RFIC 2009. IEEE. pp. 113-116. IEEE (2009)

[62] Gamm, G., Sippel, M., Kostic, M., Reindl, L.: Low power wake-up receiver for wireless sensor nodes. In: Intelligent Sensors, Sensor Networks and Information Processing (ISSNIP), 2010 Sixth International Conference on. pp. 121-126. IEEE (2010)

[63] Fraunhofer Institute: Wakeup receiver (2010), http://www.iis.fraunhofer.de/ en/bf/ic/komp/rf/hew.html

[64] Marinkovic, S.J., Popovici, E.M.: Nano-power wireless wake-up receiver with serial peripheral interface. In: IEEE Journal On Selected Areas in Communications. vol. 29, pp. 1641 - 1647 (Jan 2011)

[65] Zarlink Semiconductor Inc: Medical implantable rf transceiver mics rf telemetry (2010), http://www.zarlink.com/zarlink/ zl70102-shortform-datasheet-jun10.pdf

[66] Rules, F., Regulations: Mics band plan (2003)

[67] Jelicic, V., Magno, M., Brunelli, D., Bilas, V., Benini, L.: Analytic comparison of wake-up receivers for wsns and benefits over the wake-on radio scheme. In: Proceedings of the 7th ACM workshop on Performance monitoring and measurement of heterogeneous wireless and wired networks. pp. 99-106. ACM (2012)

[68] Zussman, G., Segall, A.: Bluetooth time division duplex - analysis as a polling system. Columbia University pp. 547-556 (2004)

[69] Farserotu, J., Gerrits, J., van Veenendaal, G., Lobeira, M., Long, J.: Csem fmuwb proposal. IEEE P802.15 Working Group for Wireless Personal Area Networks (WPANs) (2009) 
[70] Kamath, S.: Application note an092 - measuring bluetooth low energy power consumption. Texas Instruments (2010)

[71] Selvig, B.: Application note an053 - measuring power consumption with cc2430 \& z-stack. Texas Instruments (2007)

[72] Miller, M., Vaidya, N.: A mac protocol to reduce sensor network energy consumption using a wakeup radio. IEEE Transactions on Mobile Computing pp. 228-242 (2005)

[73] Marinkovic, S., Popovici, E.: Ultra low power signal oriented approach for wireless health monitoring. Sensors 12(6), 7917-7937 (2012) 